\title{
A Security Analysis Method for Industrial Internet of Things
}

\author{
Haralambos Mouratidis, Vasiliki Diamantopoulou, Member, IEEE
}

\begin{abstract}
The Industrial Internet of Things (IIoT) provide an opportunity for industries to build large interconnected systems that utilise various technologies such as personal computers, wireless devices, and sensor devices and bring together the cyber and the physical world. Such systems provide us with huge advantages but they also introduce major security challenges at both the design and runtime stages. The literature argues for the need to introduce security-by-design methods, which enable security analysis and mitigation of security threats. This paper proposes a novel security-by-design method for IIoT environments across two different levels, design/modelling and runtime/simulation. Our method supports analysis of security requirements and identification of attack paths and their integration for the mitigation of potential vulnerabilities. We demonstrate its applicability through a real case study on a critical environment from the maritime sector which demonstrates that our method helps to identify security mechanisms to mitigate attacks on critical assets.
\end{abstract}

Index Terms-Industrial Internet of Things, Security Requirements Engineering, Attack Path Discovery.

\section{INTRODUCTION}

$\mathbf{T}$ ECHNOLOGY is changing the way that we communicate, learn, and work. In the industrial sector, the number of computational components integrated into production systems, transportation and logistics is rapidly increasing, creating a large Industrial Internet of Things (IIoT). It is estimated [1] that the number of connected devices will reach more than 70 billions in 2025. Such interconnected environment can provide major benefits, such as the monitoring of the move of goods across a supply chain, from manufacturing to distribution [2]. On the other hand, appropriate security management approaches are required to ensure security of IIoT environments. A major security challenge for IIoT is analysing the connections between all different components, at physical and cyber level, (e.g., devices, infrastructures, etc.) and the potential vulnerabilities that arise from those connections [3], [4] in order to limit unathorised access and mitigate potential security attacks. For example, a device might be attacked through exploitation of a component that is connected with that device [5]-[7].

In responding to such challenge, security for IIoT must be approached in a proactive manner. Such approach requires, on one hand, the development of new systems (or the analysis of existing systems) that follow security-by-design principles, i.e. development and analysis based on security requirements engineering; and on the other hand, approaching security as a moving target where new developed attacks and new vulnerabilities can be identified and their impact on the IIoT environment is analysed. It is therefore important that we develop methods that enable us, on one hand, to identify security requirements, and model and analyse IIoT environments based on those security requirements (thus following a security-by-design approach) and on the other hand, to enable the identification of attack paths that an attacker could exploit and provide analysis of the impact that those have.

This paper makes an important contribution to the security of IIoT by proposing, to the best of our knowledge, the first security-by-design method and demonstrate its applicability through its applications to a real life IIoT scenario. In particular, the proposed method supports analysis of IIoT environments at two different levels. At the Modelling Level the method provides a process and a modelling language that supports the modelling and analysis of connections between IIoT components, their security requirements, and for existing systems, the current set of security mechanisms employed. At the Simulation Level the proposed method provides a set of algorithms that can automatically identify potential attack paths and categorise the importance of such paths. The output of the simulation level can be fed back to the modelling level to identify extra security measures to mitigate the identified attack paths.

The remainder of the paper is set out as follows: Section II discuses related work, focusing on the security challenges of IIoT and IoT, since the particular area of IIoT provides limited results, on risk management approaches and on network based attack path discovery studies. Section III presents our proposed method while Section IV illustrates its application to a case study in an IIoT environment from the maritime transport. Finally, Section V summarises the conclusions and raises issues for further research.

\section{RELATED WORK}

One of the novel aspects of our method is that it integrates three different research areas, security analysis of complex cyberphysical systems, such as the one of IIoT, risk management approaches, and network based attack path discovery approaches. For this reason, since there is no other method that combines these three areas, we focused our literature review on these three areas separately.

Security in IIoT and IoT In the demanding and challenging area of IIoT limited work that examines security issues has been identified. The authors in [8] examine and identify the peculiarities of IIoT systems, and compare them to traditional ones that affect the adaptation of existing information security concepts. The authors argue that in such systems a holistic cybersecurity concept for IIoT is required, able to address 
the various security and privacy risks at all abstraction levels. Another work that focuses on security of industrial sensors in the context of the IoT has been conducted by [9]. The authors analyse the features of the IoT and identify numerous security challenges that must be solved, such as protocol and network security services able to offer end-to-end secure communication channels and the provision of authentication mechanisms for identity management. Moreover, in order to understand how the different approaches of IoT environments can be secured, they analyse various attacker models, such as DoS, physical damage, eavesdropping, node capture, and controlling. They conclude that these different environments can create new threats and facilitate attackers, but on the other hand, can reduce the effectiveness of certain attack vectors.

We further extend our research by examining security in IoT environments, in general. This approach will give us the confidence that we have examined all the relevant aspects related with security in such environments. Hence, we identified some recent studies in this direction. More specifically, the authors in [10] have conducted an empirical investigation regarding security of IoT applications, based on two real casestudies. They identified that despite that security is considered an important value for the IoT products, however, during product development there is lack of practical consideration of security. They also highlight the importance of tailoring processes, able to address IoT-related security challenges. The authors in [11] propose an IoT security model which aims to capture security and privacy aspects, i.e. device protection, device boot, authentication, communication, device monitoring and reporting, personal data protection, and data transmission security. However, this model is presented from a theoretical perspective, and lacks providing any implementation approach. The authors in [12] mention security threats in three layers of IoT architecture. In front-end sensors and equipment, potential threats refer to unauthorised access to data, threats to the Internet and Denial-of-Service attacks. In the network layer, they identify Denial-of-Service attacks and in the back-end of IT systems they mention seven standards, i.e. privacy protection, access control, user authentication, communication layer security, data integrity, data confidentiality, and availability at any time. This work highlights the threats that are identified in any layer of the IoT architecture, but it lacks of providing specific solutions for the protection of the assets of the IoT environment against those threats.

Risk Management In attack graph generation and analysis several approaches can be found in the literature. In the direction of risk assessment and risk management, the authors in [13] present a parameterised cyber-attack path discovery method which uses constraints and depth-first search for the generation of attack paths that an administrator of a system is interested in. Another approach for attack graph generation for risk assessment is the one proposed by [14]. This method provides scalability and is based on a cut and divide method and a series of division round, while it uses depth-first search to search the smaller graphs.

Network based attack path discovery Typically graph construction takes place within a network to identify all possible attack paths that can be exploited by attackers to gain unauthorised access to the system. For instance, MulVal is a well-established enterprise network security analyser that is based on logic [15]. It models software bug interactions along with network configuration, with data supplied by an open source reporting community. Another tool for generating attack graphs can be found in [16] with the name TVA. This is a tool for topological network analysis, based on graph dependency exploitation. In [17] the authors implemented an intrusion detection system that produces a graph as output. NuSMV [18] is another model checking tool that finds vulnerabilities and generates an attack graph. The work proposed by [19] is yet another logic based approach that uses deduction to form the attack graph. Solutions that are closer to our method exist, with one found in [18] that uses a Breadthfirst search method to identify the vulnerabilities and build the attack graph. Furthermore, more recent approaches exist and offer different solutions to generate attack graphs. In [20] the authors propose a distributed approach to attack graph generation. This method is based on a multi-agent system, a virtual shared memory abstraction and hyper graph portioning to improve the performance. This method uses a Depth-first search method where the performance is improved with the use of multiple agents after a specific network size. It is also shown that in small network sizes a single threaded approach is faster. In [21] the authors propose the use of a dynamic algorithm that generates an attack graph consisting of the top $\mathrm{K}$ paths that there is a probability of being exploited.

In comparison to the above works, our work differentiates as it provides a security-by-design approach, able to identify security requirements in IIoT environments, from the early stages of the development of a system, and based on these requirements, to efficiently identify the critical attack paths that a malicious user can exploit.

\section{PROPOSED METHOD}

We propose a novel method for security modelling and analysis of IIoT environments, which supports development of secure IIoT environments at two levels, modelling and simulation. Fig. 1 illustrates the main stages of the proposed method. At the modelling level, the proposed method enables (through a modelling and analysis process) modelling of an IIoT environment component, and their dependencies (IIoT Environment Analysis) along with security related issues, such as security constraints, security threats and security mechanisms (IIoT Environment Security Modelling). At the simulation level, the method enables (through algorithms) the identification of potential attack paths to the IIoT environment along with a determination of the importance of those attack paths. The results from the simulation can then be fed back to the output of the modelling level to enable further analysis (in terms of security measures identification) and mitigation of the attacks that have been identified as important.

\section{A. Modelling Level}

The first stage (Stage 1) of the proposed method is the IIoT Environment Analysis. During that stage, the aim is to create a model of the IIoT environment, which includes all 


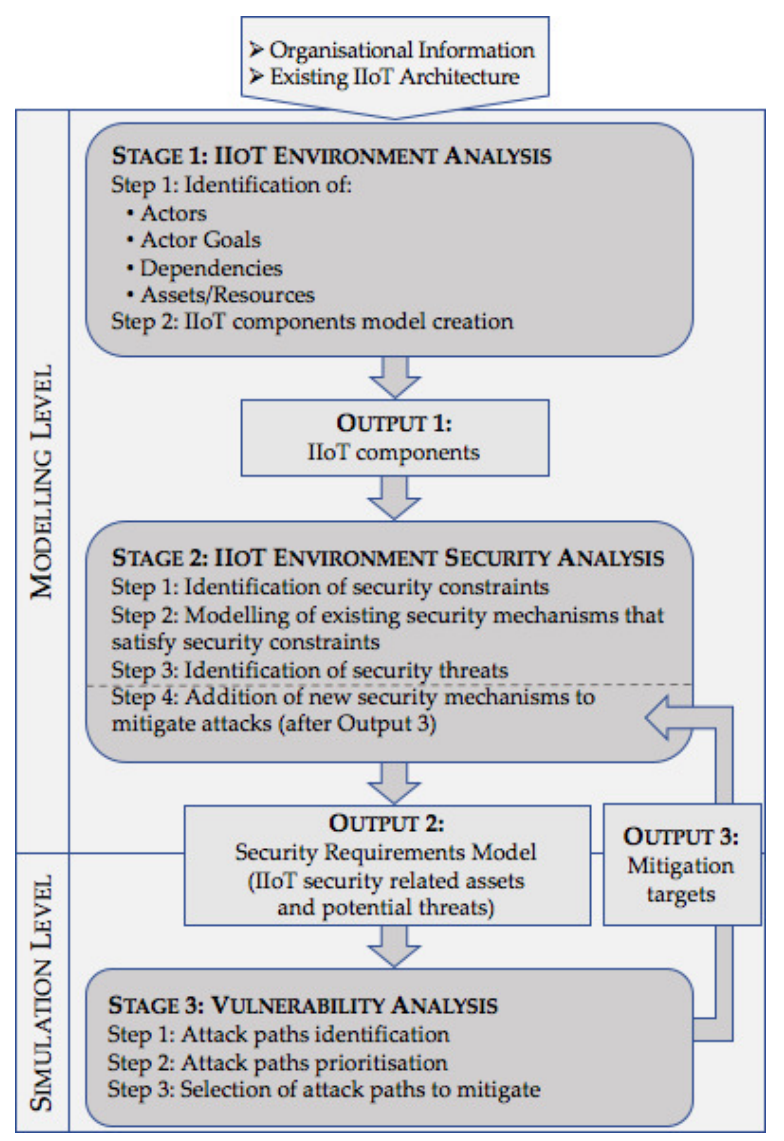

Fig. 1. Process of the proposed method

the relevant IIoT components along with their dependencies. The input for the creation of such model is information from the relevant stakeholders related to the IIoT environment, including information about components (physical, cyber and human) and how they are related. To create the model, our method is based on the Secure Tropos [22] modelling language from the security requirements engineering area. In particular, we enhance concepts of the Secure Tropos language such as actor, dependency, plan and resource to enable us to model the components and relationships between components of IIoT environments. Using that approach, each IIoT component (cyber, physical, human) is represented as an actor, which has some strategic goals (functionalities or aims), relevant plans (tasks) for achieving those goals and a set of assets (resources) required for carrying out the plans. Moreover, each actor can have a number of dependencies for goals/tasks that cannot achieve on their own. To better understand the way that a model created with our method can support the modelling of IIoT environment, consider for example a software (actor) used to control temperature (goal) to achieve that functionality the software will have a number of goals. Some of those goals can support independently, for some other functionalities will require some input from other components (physical, cyber or humans). These are modelled as dependencies. For example, the software depends on a sensor (another actor) to receive temperature readings (goal). The output of this analysis is the creation of the IIoT Components model. That model is then used as an input for Stage 2, the security modelling. At this stage, we have enhanced the language of the Secure Tropos [22] modelling language, to enable modelling of security concerns related to IIoT environments, including security requirements, security mechanisms, and security related assets. In particular, our method supports the modelling of security requirements in terms of security constraints, which are security related constraints introduced to the various components of an IIoT environment. Security constraints restrict the various goals and plans that each actor has. Going back to the temperature reading software above, a security constraint is introduced related to the receive temperature reading goal to ensure integrity of the temperature reading. For each security constraint, our method supports the identification of security mechanisms, which are measures that can ensure the satisfaction of the security constraints. An example of such mechanism for integrity is digital signatures. Moreover, the method enables the identification and modelling of potential threats. Threats are modelled at two levels, at environment level where a threat is impacting one or more goals of an actor, and on a technical level where a threat has an impact on low level mechanisms. It is worth noting that at a technical level, our approach is modelling individual threats, as well as advanced persistent threats.

Our method allows developers to represent security concerns at different levels of description, including cyber and physical levels; and to verify at the modelling stage, whether the identified security mechanisms shown in the model can satisfy the security requirements of the IIoT system [23]. It is worth mentioning that models (for both stages) are incrementally developed from a high abstraction level to a more detailed level and from a system-as-it-is to a system-tobe perspective [24]. The output of this stage is the Security Requirements Model, which is used as input for the third stage.

\section{B. Simulation Level}

The simulation level Stage 3 of the proposed method takes as input the IIoT assets that have been identified at the previous stages and, using the algorithms we have defined, performs a Vulnerability Analysis of the system that results in the identification of potential attack paths and a quantification of the importance of the various assets on such attack paths. In our method, an attack path is the identification of one or more vulnerabilities that can be exploited by attackers to gain access to specific assets and move between them in a network, thus, forming an exploitable path between the assets.

Our work is based on a previously developed algorithm found in [25], since it is highly parameterised. It takes input the location and capability of the attacker, the asset graph, and the max and propagation length (the depth of the graph that will be searched), returning the affected assets and the attack paths, or an empty graph, if no attack paths were found. These outcomes are then fed to Algorithm 2, and thus finally returning the prioritised hardware to hardware attack paths. The algorithm is particularly useful when we want to perform risk assessment in specific network parts that can be used in the IIoT domain. Also, by tweaking its parameters, a prioritisation 
of the vulnerability of these paths can be identified. This is important in IIoT due to the large number of physical, human and cyber components. Our simulation stage makes use of two algorithms: Algorithm 1 to identify the various potential attack paths, and Algorithm 2 to support analysis of certain network parts and prioritise the paths. Due to lack of space, we are not able to fully describe the algorithms of stage 3 , but we provide the pseudocodes, as shown below. Moreover, it is important to note the following: (i) The capability of the attacker could be either low, medium or high, while the location of the attacker could be local, adjacent or network based. The entry and target points specify which are the entry points that we want to assess and which are the target points. (ii) The path construction phase follows rules that belong to the propagation and allow us to determine which vulnerabilities the attacker can use to further penetrate the infrastructure to reach a predefined target or to cause multiple damage by affecting as many assets as possible from a given entry point/asset. (iii) We examine how an attacker can exploit identified cyber asset vulnerabilities in order to perform undesired actions. For every attack, a set of related weaknesses, from the Common Weaknesses Enumeration (CWE) database, and vulnerability types are defined. It is assumed that to perform this kind of attack the attacker must have access to an asset that has one or more vulnerabilities that are compatible with either the weaknesses or the type defined. (iv) The asset configuration includes information about a particular asset. For example, the name of the asset, an id, the business partner to which this asset belongs, its vulnerabilities, and attributes from the Common Vulnerabilities and Exposure (CVE) repository, such as access complexity and access vector. The entry point and the target points are specific cyber assets on which a business partner wants to focus on.

Algorithm 1: Attack path discovery

Input: Asset graph, max and propagation length, attacker location and capability

Output: Affected assets, attack paths

\#Load the graph and store it in memory

Load Asset graph from database

Create Graph data structure $\mathrm{G}$ and store the asset graph

For e in parameters entry points

If attacker location < required level of attacker location

OR attacker capability < required attacker capability

Else

Return empty graph

Get single source shortest path length

\#Create list with all no-circular paths from entry e to target $t$

For entry point e

For target point $\mathrm{t}$

If exists no-circular path from e to $t$

Add path to list

\#Search the graph up to the specified length

Get all paths in the graph $G$ from entry e to target $t$ that are up to the pre-specified path length

For the size of paths found

Add paths to attackpaths list

Add affected assets to affectedassets list
Return attack paths, affected assets

Algorithm 2: Prioritised hardware to hardware attack path discovery

Input: Affected assets, attack paths (Algorithm 1 output)

Output: Prioritised hardware to hardware attack paths

Load affected assets

Load attack paths

For each attack path

Load the total number of vulnerabilities

For each vulnerability

calculate the importance of each vulnerability using equation 1 (Step 1)

calculate the sum of the importance of each vulnerability using equation 2 (Step 2)

Apply equation 3 to get the average vulnerability importance (Step 3)

Load from the database the administration importance of input and output hardware asset in the attack path (Step 4)

Create an attack path that contains only the hardware to hardware asset

Add attack path from above to [prioritised hardware to hardware attack paths] list

Apply equation 4 to each of the hardware to hardware attack paths (Step 5)

Convert [prioritised hardware to hardware attack paths] values in range 1 to 100 (Step 6)

return prioritised hardware to hardware attack paths

The summary of the steps and the respective (where applicable) equations are shown below. Step 1: $0.6 *$ Impact $+0.4 *$ Exploitability (1)

Step 2: $\sum_{1}^{n}$ value from equation 1 (2)

Step 3: $\frac{\text { ValuefromStep } 2}{\text { NumberofVulnerabilities }}$

Step 4: Fixed numerical value assigned from the administrator when the asset is added to the database

Step 5: $0.6 *$ value from Step $4+0.4 *$ value from Step 3 (4)

Step 6: Convert to percentage

It is worth noting that for the coefficients in the above formulas we consider the widely used values found in the literature from CVSS to calculate the base score. Moreover, impact and exploitability are values that are standard on CVE (see an example at https://nvd.nist.gov/vuln/detail/CVE-20151769). Impact refers to the severity of a potential exploitation and exploitability refers to how difficult is to exploit an asset. Both these take values from 1-10. This means the proposed method calculates risk based at how many CVEs might exist at the relevant hardware, the sum of the scores of the CVEs and the value of the administrator. The benefit of our approach is that we also include the admin value on the calculation, which our research indicated that this is important. 
The selection of the attack paths to mitigate, as resulted from the execution of Algorithm 2, is then fed back to Step 4, in order to identify new security mechanisms to mitigate the identified attack paths. In particular, the selection of the attack paths is based on the administrator(s) who can set a threshold for the selection of attack paths that will be mitigated. Once the relevant attack paths are identified, the mitigation targets (in terms of assets relevant to those paths) are further analysed, as part of Step 4 of the modelling level stage 2 analysis, involving the identification of extra security mechanisms for those assets to mitigate vulnerabilities that have identified by the simulation results and the verification at modelling level of those extra security mechanisms.

\section{CAse Study}

To better illustrate the applicability of the proposed method we present how it is applied in an IIoT environment from the maritime transport, which is considered as the backbone of international trade and a key engine driving globalisation [26]. In a usual maritime port environment there are a series of supply chain services, such as: i) Container Cargo Management, ii) Vehicles Transport, iii) Liquefied Natural Gas Transport, and iv) Bulk/General Cargo Transport. Due to space limitations, we are focusing on the Vehicles Transport supply chain service, which is considered as a massively complex IIoT system with numerous components, including shippers, IT systems, and transport operators that involve the shipment and the reception of various types of vehicles and equipment such as trucks, vans, truck trailers, threshing machines, etc. This service is considered as a relatively long and complicated process that involves domestic and international transportation, warehouse management, order and inventory control, materials handling, import/export facilitation, and information technology. In this framework, it is clear that the vehicles transport affects many sectors along the supply chain. Focusing again on the Vehicles Transport supply chain service, it can be broken down to a number of processes that involve several physical (docking of the ship, stevedoring, loading, unloading, storage, transportation, inspection, etc.) and cyber (pre-arrival notifications, customs clearance documentation management, International Ship and Port Facility Security declaration, etc.) operations, interconnections and assets.

We apply the method presented in Section III, to model security requirements of the vehicle transport supply chain service, analyse relevant security mechanisms and threats, and identify and mitigate attack paths. In this case study we focus on one system, the one of Port Authority and their dependencies with the Port Community system and with the Supervisory Control and Data Acquisition (SCADA) system. Following the stages of the proposed method, as illustrated in Fig. 1, we split the application to two levels: modelling and simulation. We describe those in the next two subsections.

\section{A. Modelling Level}

The first stage of the modelling level includes the IIoT environment analysis, which involves identification of actors, their goals and the dependencies among these actors, and relevant assets, as illustrated in Fig. 2, and explained below. In that figure, actors are represented as pink circles, goals as green rounded rectangles, resources as yellow rectangles, and plans as blue hexagons. Dependences are represented as arrows from the depender actor to the dependee one. Actor boundaries are represented as dashed pink rounded rectangles.

IIoT Environment Analysis: In the vessel docking process, the Ship depends on the Ship Agent to obtain docking arrangement. The actor Ship Agent uses the e-services offered by the Customs Authority. Consequently, Ship Agent depends on Customs Authority for obtaining "Customs Clearance Approval" document. Next, Customs Authority depends on the Integrated Customs Information System to obtain "Customs Clearance Approval" data. The Customs Clearance Approval is the documented permission given by the Customs to import or export vehicles and proves that all Customs duties have been paid and shipment procedures have been approved. This document has to be submitted by the Ship Agent to the Port Authority. Once the Ship Agent obtains the Customs Clearance Approval, they submit it to the Port Authority. So, Port Authority is depended on Ship Agent in order to receive the "Custom Clearance Approval".

After the submission of the document, the Ship Agent is dependent on the Port Authority in order to use their services for the vessel, such as, mooring, lacing, assigning risk assessment processes, weather conditions, navigational warnings, procedures for communication failure, fenders, personnel (truckers for transferring the vehicles from the ship to storage area, etc.). Ship Agent requests the aforementioned services for the vessel from the Port Authority and this process is performed via the actor Port Community System, an electronic platform which connects the multiple systems operated by a variety of organisations involved in the port's supply chain. Thus, Port Authority is depended on Port Community System to i) receive the necessary information for the vessel docking, and ii) provide e-port services. Actually, this system acts as a centralised system that supports a variety of eport services aiming at facilitating the secure and efficient electronic exchange and management of information between the involved public and private stakeholders, and promoting the automatisation and the smooth operation of the port and logistics processes through a single request submission.

In this context, the Port Community System, in order to check the "Custom Clearance Approval" document authentication by data verification services, depends on the Integrated Customs Information System, requesting the necessary information via an Electronic Data Interchange (EDI) system, and reports the result. The Port Community System depends on the "Marine Traffic web platform" to obtain information on vessels position and the Arrival/Departure timetable. This information is obtained via the e-tracking (Marine Traffic Internet AIS) services, hosted by the "Marine Traffic web platform". Then, once all relevant formalities have been arranged by the authorised entities (i.e. docking clearance submission), and the port's equipment (yard tractors, forklifts with the auxiliary equipment like sensors, etc.) and port personnel is available to unload the vehicles and store them to the port's facilities, the Ship Agent informs the Ship about the docking 


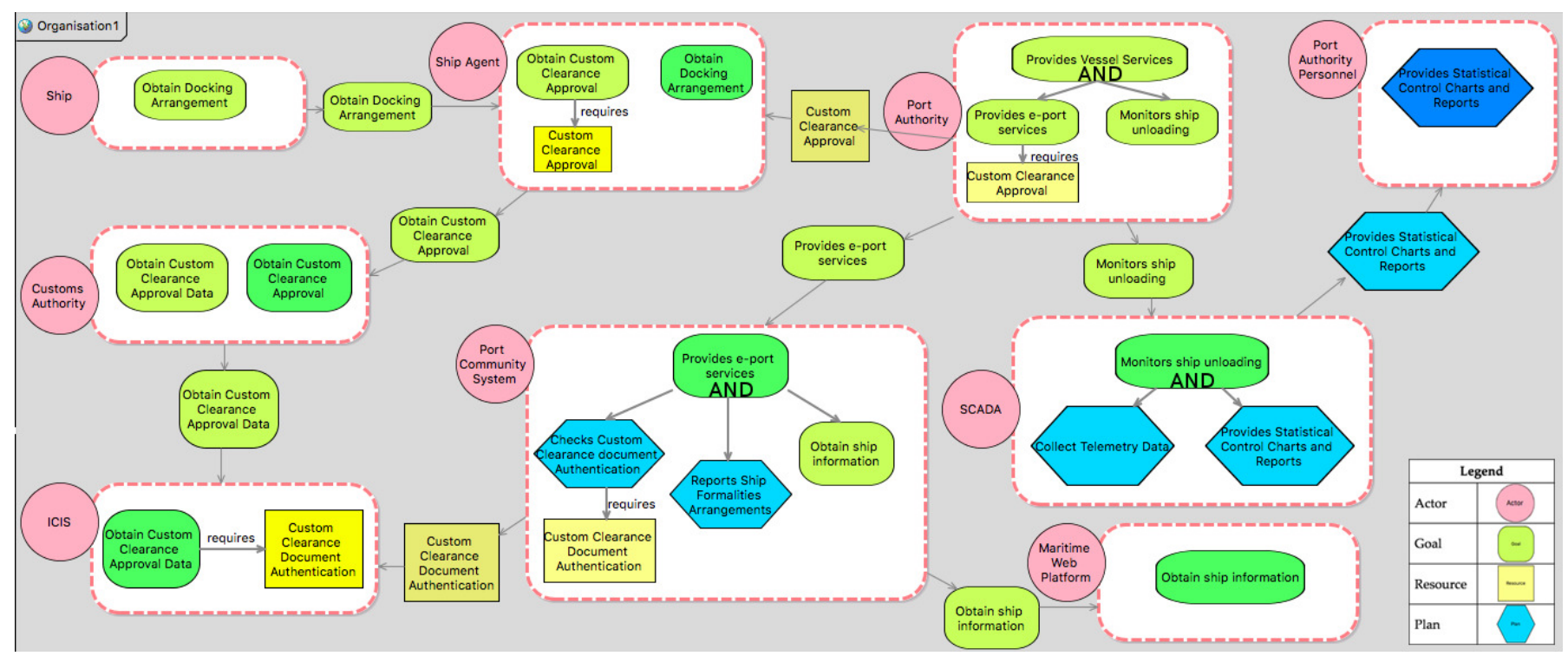

Fig. 2. IIoT Environment Analysis

arrangements. In the unloading vehicles process, when the vessel arrives at the port, a SCADA system's goal is to monitor vehicles' unloading from the vessel, which is performed by yard tractors and forklifts with their auxiliary equipment. The Port Authority depends on the SCADA system to i) monitor the vessel unloading and ii) gather data from distributed sensors and instruments. SCADA system analyses and displays information improving the performance of vehicles shipping operations, reducing waste of time and providing cost saving.

IIoT Environment Security Analysis: In Stage 2 the method supports identification of security constraints, relevant security mechanisms and security threats. For illustration purposes, we focus the rest of the analysis on the Port Authority actor, which is responsible for providing vessel services, such as e-port services and for monitoring ship unloading, as shown in Fig. 3. In that figure, red octagons represent security constraints, while red hexagons with parallel lines represent security mechanisms. This is a typical IIoT environment that includes human, cyber, and physical components. In particular, the Port Authority depends for the realisation of its two main goals, on the Port Community System and the SCADA system.

We focus on the SCADA system, which is a computer-based system that monitors and controls port industrial operations. The ultimate goal of the SCADA system is to "monitor ship unloading", and this goal is achieved through two plans, the "collection of telemetry data" and the "provision of statistical control charts and reports". Following the Identification of security constraints (Stage 2, Step 1) of our method, we elicit security requirements in the form of security constraints. For our case study, the two plans of the SCADA system, namely Collect telemetry data and Provide statistical control charts and reports are restricted by the following security constraints: Integrity, Non-repudiation, Confidentiality and Availability. For each one of them we identify one or more security mechanisms that support their satisfaction. Taking the Integrity security constraint as an example, in IIoT environments in- tegrity of the data should be preserved for protecting the data against a sabotage, which might lead to unnoticed loss of product quality and increased use of resources. Therefore, to satisfy such security constraint the system includes Encryption and Decryption. Similar analysis takes place for the rest of the security constraints, where the various mechanisms that exist in the system are modelled, as partially shown in Fig. 3.

Moreover, our analysis of the Collect telemetry data plan has resulted in the identification of 38 assets, including Arduino Microcontroller, Programming Logic Controller, Remote Terminal Unit, Remote Control Station, Servers, Laptops, and Mobile Devices. On the other hand, following the identification of security threats step of the method, several threats that impact the Collect telemetry data plan have been identified, such as Code Overflow, Malicious Code Execution, Denial-of-Service, Man-in-the-Middle, Keylogging, Obtain Privilege, Malware, and Phishing. For each threat we indicate the impact it has on one or more areas of the system. For example, Malware has an impact on the Collect Telemetry Data plan, due to the possibility of an attacker exploiting the Programming Logic Controller of the SCADA system using malware. Our approach is not limited to generic types of malware, but can also be applied in cases of Advanced Persistent Threats (APT), which utilise any of the Threats depicted in Figure 3 (e.g., the Stuxnet Worm [27]). The above assets and threats, together with the security constraints, are used as input for the vulnerability analysis stage.

\section{B. Simulation Level}

During the Vulnerability Analysis stage, we have executed Algorithm 1 to identify potential attack paths. The execution has resulted in the identification of 78 attack paths, which contain both hardware and software assets. These assets are then fed into the second Algorithm, where it only identifies hardware to hardware paths and generates the Importance percentage of each path, which is used in order to prioritise the 


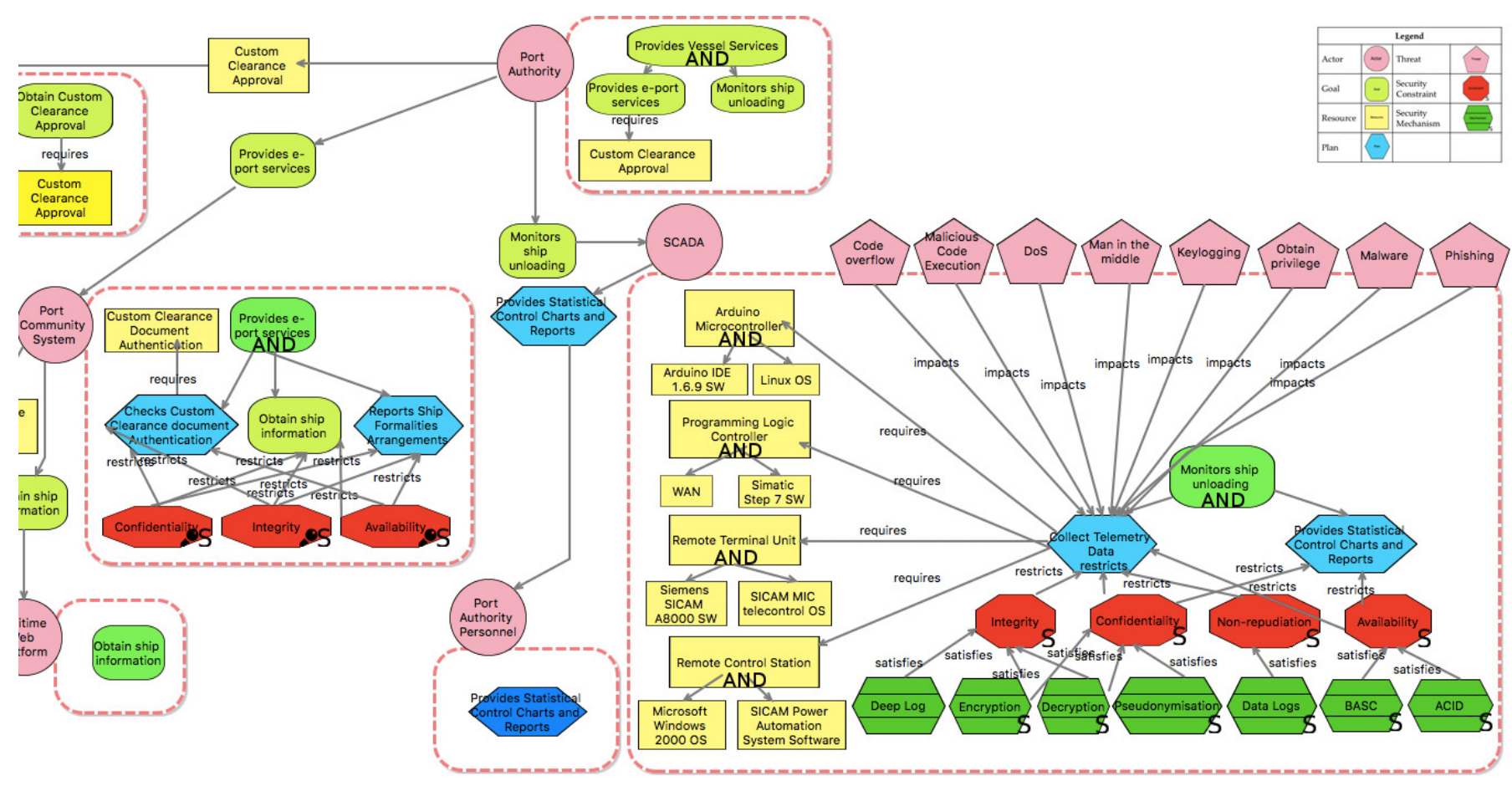

Fig. 3. IIoT Environment Security Analysis

sequence of the mitigation of each path. That means that the attack paths with the highest importance has to be mitigated first, as their damage in a potential cyber attack will cause more critical problems to the system. It is worth mentioning that for the identification of the threshold, we take into account experts and stakeholders advice, who indicate, according to the data they are provided, a relevant percentage, e.g., to proceed with the mitigation of the first $10 \%$ of the identified attack paths. Our analysis has identified 10 paths which have importance $100 \%$. Consequently, these paths need to be mitigated first. The assets that are included in these paths are presented in Table I. The next step of Vulnerability Analysis involves the selection of attack paths to mitigate. These attack paths are then fed back to Step 4 of the IIoT Environment Security Analysis, in order to identify new security mechanisms to mitigate them. For example, for attack path 22, to mitigate attacks that affect the Integrity of the identified assets, we enhance the security mechanisms to include Digital Signature and Data Obfuscation, while to mitigate attacks that affect Availability we enhance the security of the system to include mechanisms, such as Mirroring Servers and Update Device Firmware. Each of them supports specific vulnerabilities identified during our analysis, for example the Update Device Firmware security mechanism protects the Router asset of attack path 22 from vulnerability CVE-2017-5521.

The application of our work to the above case study have illustrated that the main challenges in terms of modelling and analysing security issues in IIoT environments are related to the need to understand, model and analyse security at various abstraction levels, and across the multifaceted architecture that such environments demonstrate. This helped us to better design the process of the proposed method to enable analysis
TABLE I

ATTACK PATHS WITH THE HIGHEST IMPORTANCE

\begin{tabular}{|c|c|}
\hline Path & Affected assets \\
\hline 8 & rver, Router, Remote Control Station, MS \\
\hline 18 & [Laptop, Router, Linux Server, Desktop, MS Server] \\
\hline 19 & $\begin{array}{l}\text { [Laptop, Router, Remote Control Station, MS } \\
\text { Server] }\end{array}$ \\
\hline 21 & $\begin{array}{l}\text { [Laptop, Router, Remote Control Station, MS Server, } \\
\text { Linux Server] }\end{array}$ \\
\hline 22 & $\begin{array}{l}\text { [Laptop, Router, Remote Control Station, Mobile } \\
\text { device, MS Server] }\end{array}$ \\
\hline 23 & [Laptop, Router] \\
\hline 30 & $\begin{array}{l}\text { [Laptop, Router, Remote Control Station, MS } \\
\text { Server] }\end{array}$ \\
\hline 39 & [Laptop, Router, Linux Server, Desktop, MS Server] \\
\hline 40 & $\begin{array}{l}\text { [Laptop, Router, Remote Control Station, MS } \\
\text { Server] }\end{array}$ \\
\hline 43 & $\begin{array}{l}\text { [Laptop, Router, Remote Control Station, Mobile } \\
\text { device, Server] }\end{array}$ \\
\hline
\end{tabular}

across such different abstractions levels. For example, the modelling stage now includes modelling and analysis at two different abstraction levels, i.e. environment and security, rather than one level that was the original approach. Moreover, the application of our work to the case study has identified the need to focus the second algorithm to the prioritisation of the hardware to hardware attacks and the need to include the administrator input. Last but not least, the practical application of our work revealed the need to introduce step 4 of stage 2 
of the modelling level. In the original version of the method, the output from the simulation was used as input to step 1 of stage 2 . However, this resulted in unnecessary analysis and it was time consuming as the output of the simulation does not change the security requirements.

\section{Conclusions}

We propose a novel security method for security modelling and analysis of such environments. This method aims to support the development of secure IIoT environments at the modelling and simulation level. As future work, we plan to examine the applicability of our proposed security analysis method to other complex environments, such as connected cars and homes, and wearable devices. This will allow us to understand in depth the challenges that these environments face, the peculiarities that have to be addressed, in order to protect the exchanged information of the distributed devices and the service provision of all relevant actors, limiting the number of incidents that can affect negatively the entire IIoT environment. We also aim to extend our work by including privacy requirements during the modelling and the analysis of such systems. Through this extension we will be able to preserve privacy on users' data and identify the critical paths that might have impact on users' privacy requirements, such as anonymity, unlinkability, unobservability, undetectability, and so on. Finally, we plan to enhance the simulation level with game theoretic approaches, so we can better identify mitigation techniques for the identified attack paths.

\section{REFERENCES}

[1] Statista. (2017) Internet of things (IoT) connected devices installed base worldwide from 2015 to 2025 (in billions). [Online]. Available: https://www.statista.com/statistics/471264/ iot-number-of-connected-devices-worldwide/

[2] L. Da Xu, W. He, and S. Li, "Internet of things in industries: A survey," IEEE Transactions on industrial informatics, vol. 10, no. 4, pp. 22332243, 2014.

[3] A. Jacobsson, M. Boldt, and B. Carlsson, "On the risk exposure of smart home automation systems," in Future Internet of Things and Cloud (FiCloud), 2014 International Conference on. IEEE, 2014, pp. 183-190.

[4] V. Mookerjee, R. Mookerjee, A. Bensoussan, and W. T. Yue, "When hackers talk: Managing information security under variable attack rates and knowledge dissemination," Information Systems Research, vol. 22, no. 3, pp. 606-623, 2011.

[5] R. H. Weber, "Internet of things-new security and privacy challenges," Computer law \& security review, vol. 26, no. 1, pp. 23-30, 2010.

[6] A. Cui and S. J. Stolfo, "A quantitative analysis of the insecurity of embedded network devices: results of a wide-area scan," in Proceedings of the 26th Annual Computer Security Applications Conference. ACM, 2010, pp. 97-106.

[7] S. Sicari, A. Rizzardi, L. A. Grieco, and A. Coen-Porisini, "Security, privacy and trust in internet of things: The road ahead," Computer Networks, vol. 76, pp. 146-164, 2015.

[8] A.-R. Sadeghi, C. Wachsmann, and M. Waidner, "Security and privacy challenges in industrial internet of things," in Design Automation Conference (DAC), 2015 52nd ACM/EDAC/IEEE. IEEE, 2015, pp. 1-6.

[9] C. Alcaraz, R. Roman, P. Najera, and J. Lopez, "Security of industrial sensor network-based remote substations in the context of the internet of things," Ad Hoc Networks, vol. 11, no. 3, pp. 1091-1104, 2013.

[10] A. N. Duc, R. Jabangwe, P. Paul, and P. Abrahamsson, "Security challenges in iot development: a software engineering perspective," in Proceedings of the XP2017 Scientific Workshops. ACM, 2017, p. 11.

[11] M. S. Moganedi and J. S. Mtshweni, "Beyond the convenience of the internet of things: Security and privacy concerns," 2017.

[12] J. S. Kumar and D. R. Patel, "A survey on internet of things: Security and privacy issues," International Journal of Computer Applications, vol. 90, no. 11, 2014.
[13] N. Polatidis, M. Pavlidis, and H. Mouratidis, "Cyber-attack path discovery in a dynamic supply chain maritime risk management system," Computer Standards \& Interfaces, 2017.

[14] J. Lee, H. Lee, and H. P. In, "Scalable attack graph for risk assessment," in Information Networking, 2009. ICOIN 2009. International Conference on. IEEE, 2009, pp. 1-5.

[15] X. Ou, S. Govindavajhala, and A. W. Appel, "Mulval: A logic-based network security analyzer." in USENIX Security Symposium. Baltimore, MD, 2005, pp. 8-8.

[16] S. Jajodia, S. Noel, and B. O'Berry, "Topological analysis of network attack vulnerability," in Managing Cyber Threats. Springer, 2005, pp. 247-266.

[17] P. Ning and D. Xu, "Learning attack strategies from intrusion alerts," in Proceedings of the 10th ACM conference on Computer and communications security. ACM, 2003, pp. 200-209.

[18] P. Ammann, D. Wijesekera, and S. Kaushik, "Scalable, graph-based network vulnerability analysis," in Proceedings of the 9th ACM Conference on Computer and Communications Security. ACM, 2002, pp. 217-224.

[19] X. Ou, W. F. Boyer, and M. A. McQueen, "A scalable approach to attack graph generation," in Proceedings of the 13th ACM conference on Computer and communications security. ACM, 2006, pp. 336-345.

[20] K. Kaynar and F. Sivrikaya, "Distributed attack graph generation," IEEE Transactions on Dependable and Secure Computing, vol. 13, no. 5, pp. 519-532, 2016

[21] K. Bi, D. Han, and J. Wang, "K maximum probability attack paths dynamic generation algorithm," Computer Science and Information Systems, vol. 13 , no. 2 , pp. $677-689,2016$.

[22] H. Mouratidis and P. Giorgini, "Secure tropos: a security-oriented extension of the tropos methodology," International Journal of Software Engineering and Knowledge Engineering, vol. 17, no. 02, pp. 285-309, 2007.

[23] H. Mouratidis, "Secure software systems engineering: the secure tropos approach," JSW, vol. 6, no. 3, pp. 331-339, 2011.

[24] V. Diamantopoulou, M. Pavlidis, and H. Mouratidis, "Evaluation of a security and privacy requirements methodology using the physics of notation," in ESORICS SECPRE. Springer, 2017.

[25] N. Polatidis, E. Pimenidis, M. Pavlidis, and H. Mouratidis, "Recommender systems meeting security: From product recommendation to cyber-attack prediction," in International Conference on Engineering Applications of Neural Networks. Springer, 2017, pp. 508-519.

[26] "United nations conference on trade and development (unctad) 2012 annual report"

[27] S. Karnouskos, "Stuxnet worm impact on industrial cyber-physical system security," in IECON 2011-37th Annual Conference on IEEE Industrial Electronics Society. IEEE, 2011, pp. 4490-4494.

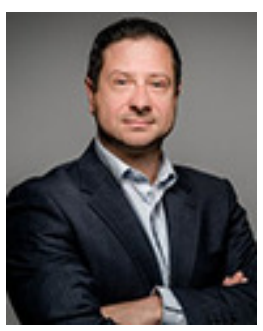

Haralambos Mouratidis is Professor of Software Systems Engineering at the School of Computing, Engineering and Mathematics, and Director of the Centre for Secure, Usable and Intelligent Systems (CSIUS) at the University of Brighton, UK. His research interests lie in the area of secure software systems engineering, requirements engineering, and information systems development. He has published more than 140 papers (h-index 28) and he has secured more than $£ 3 \mathrm{M}$ to support research and knowledge transfer.

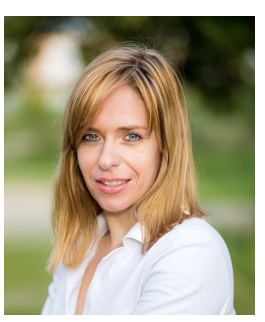

Vasiliki Diamantopoulou is Post Doctoral Researcher at the Department of Information and Communication Systems Engineering at the University of the Aegean and a Senior Researcher at the Department of Digital Systems at the University of Piraeus, Greece. She has worked as a Research Fellow at the School of Computing, Engineering and Mathematics at the University of Brighton, UK. The focus of her publications is on Privacy and Security of Information Systems, eGovernment and Interoperability Frameworks, and eBusiness and Innovation 